\title{
Technologies for Advanced Induction Accelerators
}

M.A. Hernandez, G. Kamin, R. Hanks, W. Sharp, G. Duncan, C. Sangster, L. Ahle, A. Friedman, D. Grote, D. Autrey, E. Halaxa, C. Williams

\section{April 20, 2000}

U.S. Department of Energy

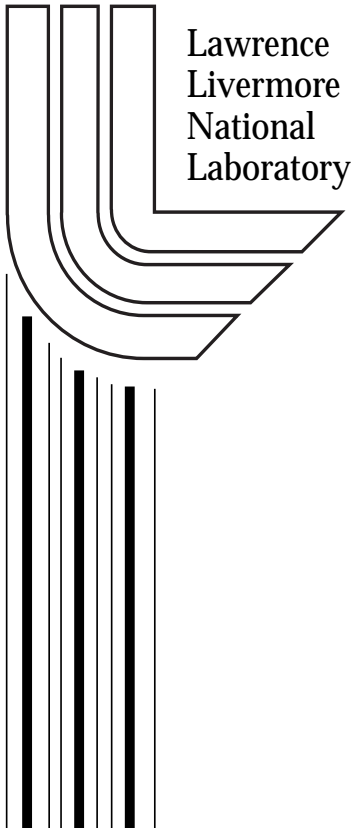




\section{DISCLAIMER}

This document was prepared as an account of work sponsored by an agency of the United States Government. Neither the United States Government nor the University of California nor any of their employees, makes any warranty, express or implied, or assumes any legal liability or responsibility for the accuracy, completeness, or usefulness of any information, apparatus, product, or process disclosed, or represents that its use would not infringe privately owned rights. Reference herein to any specific commercial product, process, or service by trade name, trademark, manufacturer, or otherwise, does not necessarily constitute or imply its endorsement, recommendation, or favoring by the United States Government or the University of California. The views and opinions of authors expressed herein do not necessarily state or reflect those of the United States Government or the University of California, and shall not be used for advertising or product endorsement purposes.

This work was performed under the auspices of the U. S. Department of Energy by the University of California, Lawrence Livermore National Laboratory under Contract No. W-7405-Eng-48.

This report has been reproduced directly from the best available copy.

Available to DOE and DOE contractors from the

Office of Scientific and Technical Information

P.O. Box 62, Oak Ridge, TN 37831

Prices available from (423) 576-8401

http://apollo.osti.gov/bridge/

Available to the public from the National Technical Information Service

U.S. Department of Commerce 5285 Port Royal Rd., Springfield, VA 22161

http://www.ntis.gov/

OR

Lawrence Livermore National Laboratory Technical Information Department's Digital Library http://www.llnl.gov/tid/Library.html 


\section{Technologies for Advanced Induction Accelerators \\ LDRD Tracking Code Number 97-ERD-086 \\ Final Report}

\section{Principal Investigators:}

Maurice A. Hernandez, George Kamin, Roy Hanks, William Sharp, Greg Duncan

Co-investigators:

Craig Sangster, Larry Ahle, Alex Friedman, Dave Grote, Daryl Autrey, Erni Halaxa, Carl Williams

Contact:

Maurice Hernandez

(925) 422-9392

Mail Stop L-874

hernandez10@1lnl.gov 


\section{Introduction}

To harness fusion energy is one of today's greatest technological challenges, and one well worth pursuing. Success in the development of fusion power would result in a virtually inexhaustible source of energy. The fusion reaction, the process that powers the sun and the stars, can be duplicated on Earth. However, to date these fusion processes have been the products of largescale experimental efforts. We have yet to achieve fusion in a manner that is cost effective and efficient enough to be applied in a commercial reactor. Lawrence Livermore National Laboratory (LLNL) has been centrally involved in the Nation's inertial confinement fusion (ICF) program for over 25 years. Much of the focus of the LLNL ICF Program has been the well-known effort to develop high power, short wavelength laser drivers to create the conditions necessary for the fusion process. But the ICF Program has also been investigating, in collaboration with Lawrence Berkeley National Laboratory (LBNL), the potential of heavy-ion accelerators as possible drivers. The objectives of the Laboratory Directed Research and Development (LDRD) project described in this report have been to develop some of the enabling technologies necessary for this type of heavy-ion fusion (HIF) driver. In particular, to apply adaptive control to the problem of tailored acceleration and steering of a pulsed ion beam.

\section{The Small Recirculator}

A 1991 study $^{1}$ showed that a recirculating ion beam accelerator (or recirculator) would be a promising candidate for a cost-effective driver for inertial fusion energy. Its promise is derived from the fact that the beam passes multiple times through each accelerating component. Thus, fewer and more effective accelerating elements, as well as fewer focusing magnets, are required. This leads to cost savings relative to a linear accelerator which, of course, uses each accelerating core and focusing quadrupole only once per pulse. In employing the recirculator concept, we can also avoid beam resonance effects by using an active control system to rapidly ramp the bending field, and rapidly accelerate the beam (since the oscillating frequency of the particles in the confining field is rapidly changing and hence is not resonant). This leads to the further advantage that much larger currents can be transported than are possible in a conventional synchrotron. To explore the possibilities of this accelerator concept, the LLNL HIF Program began the design and construction of a small, scaled recirculator (Figure 1).

The recirculator requires the integration of a number of complex components. State-of-the-art, high repetition rate and highly variable pulse modulators are required to drive the induction cores. The injector modulators must be capable of producing precise voltage pulses to reduce the current modulation and achieve the required beam reproducibility. For beam steering, we must drive the bending dipoles with a highly stable pulser, capable of ramping up the dipole voltage as the accelerated beam gains both kinetic and electrical energy. These components must be combined with sophisticated diagnostics and control systems.

In addition to this increased complexity, realization of a working recirculator enters a regime in which the total path length traversed by the beam is greater, the repetition rate of the induction modules is higher, and the dynamics of transporting beams around the bends are unexplored. These new and unexplored regimes make the development path leading to a small, scaled recirculator ground breaking and rich in potential scientific discovery and technological innovation. Through the three years of this project, we were able to achieve progress in several technology development areas. 


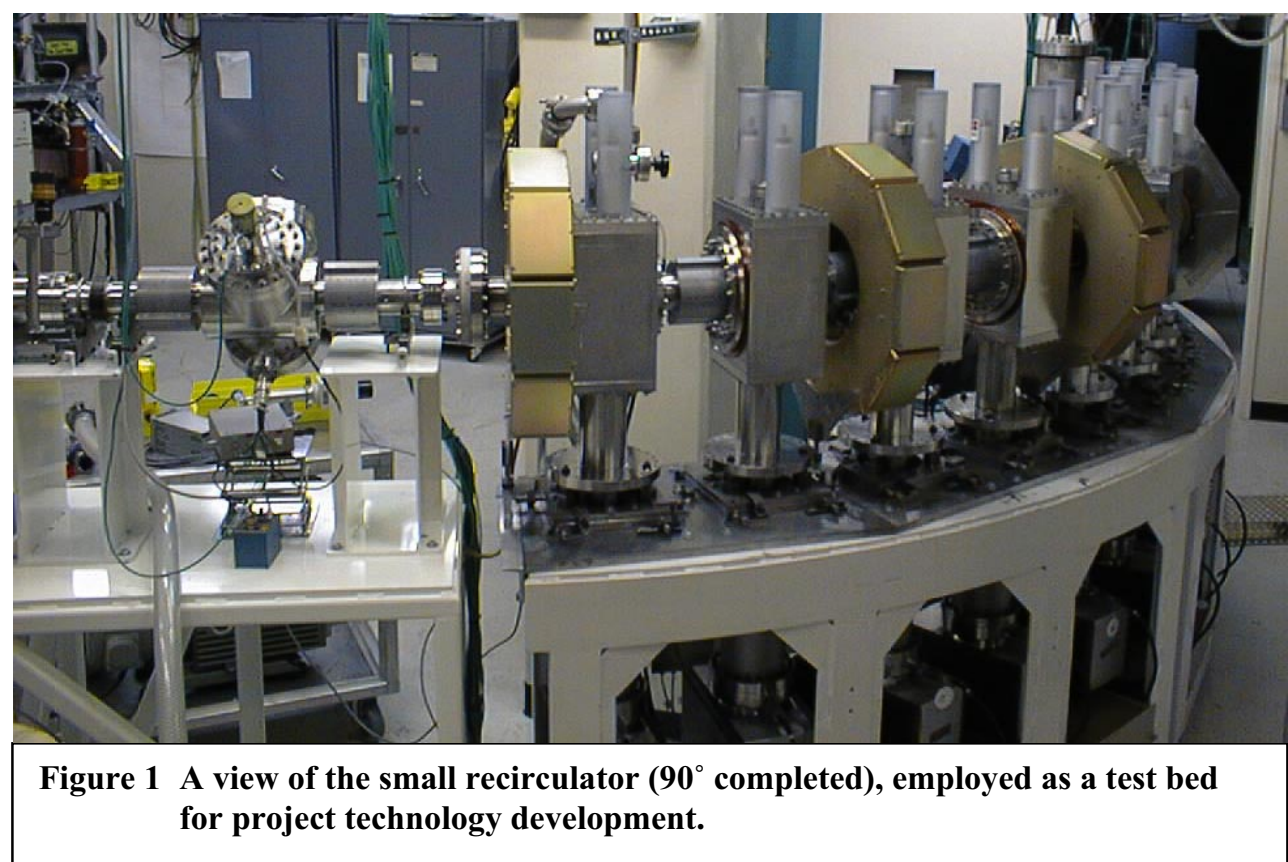

When first proposed, this project's objectives were designed to take advantage of the progressive deployment of the small recirculator, which was expected to have a $180^{\circ}$ configuration by October 1998, with a full ring completed by October 1999. Unfortunately, HIF Program priorities shifted in such a way that budget cutbacks caused the ring to be only half constructed by the end of this project (September 1999), and even then, was only able to execute beam acceleration through one induction core module. Despite the change in the expected availability of the recirculator as a project testbed, we are able to report notable achievements in various areas, including beam transport modeling, injector pulse modulators, dipole pulser development, induction core accelerator modulators, and insertion and extraction module design.

\section{Beam Transport Modeling}

During the first two years of the project we carried out a series of coordinated simulations designed to further our understanding of the beam transport dynamics. The level of precision required for successfully transporting the beam around the recirculator factors into the specifications (and cost) for many system components. Through modeling, we determined the relative effects on beam quality of errors in the injector, acceleration, steering, and sensor subsystems. We then focused our development efforts on the most critical areas. We also were able to evaluate the effectiveness of proposed control algorithms, and refine the algorithms to optimize the beam quality.

Other modeling efforts focused on refinement and optimization of the steering algorithms to minimize beam disturbance and achieve more efficient convergence. Several modeling runs were completed using varying probe configurations. The variations involve the designation of monitoring versus steering probes (both location and number), and the level of steering correction voltage applied. Relatively small steering corrections require a larger number of steering probes (which equates to higher fabrication costs), while employing fewer steering probes requires larger corrections at each probe (causing more beam disturbance). 
Early modeling results pinpointed the importance of initial (injected) beam quality, and identified the injector subsystem as a problem area. The voltage pulse produced by the original injector pulser created an ion beam with small, inherent errors. Subsequent (modeled) beam acceleration magnified the errors, creating instabilities that would make beam control more difficult. Because of these findings, an effort was initiated to re-design the injector pulser. The results of this design effort are covered later in this report.

Our simulation strategy called for sensing the position of a pulse, then applying corrections to subsequent pulses. This is as opposed to attempting to apply corrections to the same pulse on successive laps around the recirculator (a much more difficult implementation prospect). Matched centroid positions were calculated, with position and velocity corrections applied at succeeding bending dipoles. We then verified the corrections at subsequent sensors. Simulations were carried out assuming error free sensors. In practice, we anticipate that sensor errors will be small compared to cumulative errors in the steering components.

Random errors were introduced in bend strength (simulating dipole pulser errors), bend and quadrupole alignment, and beam characteristics (stability, oscillations, energy, uniformity, etc.). Error magnitudes on the order of $1 \%$ to $2 \%$ were found to be sufficient to cause the beam to impact the accelerator tube wall. Applying corrections as described in the previous paragraph demonstrated the ability to control the beam centroid within $\pm 6 \mathrm{~mm}$.

During FY97 and FY98, we studied the relative merits of two fundamental steering methods, which will help determine the best configuration for steering modules on the small recirculator. The first method calls for correcting errors in the beam centroid transverse position $X$ and velocity $X^{\prime}{ }_{-} d X / d s$ in the accelerator plane. Centroid trajectory in a particular bend section is dependent on the momentum error $p / p=\left(1-p_{0} / p\right)$ as well as on the initial values of $X$ and $X$ '. Here, $p=\ldots M c$ is the actual momentum of an ion with mass $M$, and $\left.p_{0}=F x_{-} /{ }_{-} c\right)$ is the design momentum for a dipole with a bend radius _ and a nominal bending force of $F x$, given by qeEx for electric dipoles. In these expressions for $p$ and $p_{0}$, $_{-}$is the beam longitudinal velocity scaled by the speed of light $c$, and ${ }_{-}=(1-)^{-1 / 2}$ is the Lorentz factor.

Briefly, the steering algorithm uses the linear transport matrices for the idealized lattice to estimate $X, X^{\prime}$, and $p / p$ through the steering module, consistent with measured beam-position data. The same matrices are used to calculate matched values of $X$ and $X$ ' at the second steering dipole. The needed steering corrections are then calculated using a "thin lens" approximation, modeling corrections as instantaneous changes in $X^{\prime}$ ' occurring at the midpoint of the steering dipoles. The kick at the first dipole is calculated to place the beam centroid at the matched $X$ position as it reaches the second dipole, and the kick at the second dipole is chosen to change $X^{\text {, }}$ to the matched value.

There are a great many other factors to consider in employing this steering method. A full discussion of these and the modeling results obtained from this method are given in Reference [2]. This method tends to impart larger "kicks" to the beam using relatively fewer steering modules.

The second method is based on solving for the required steering corrections using simultaneous equations designed to minimize functionals based on measured beam displacements and dipole voltages. An example functional is shown below, in which $W_{1}$ and $W_{2}$ are arbitrary weighting factors, $0<f_{1}, f_{2} \leq 1$ are arbitrary functions, and $l_{1}$ and $l_{2}$ are arbitrary powers. 


$$
F\left(X_{m}, V_{x n}\right) \ldots W_{1}{ }_{m=1}^{M} \frac{\left|X_{m}\right|}{f_{1} R} \sqrt[V]{ }^{l_{1}}+W_{2}{ }_{n=1}^{N}-\frac{\left|V_{x n}\right|}{f_{2} V_{\max }} \sqrt[V]{ }
$$

For $l_{1}, l_{2} \geq 2$, this functional remains small for displacements $<f_{1} R$ and voltages $<f_{2}$, where $R$ is the beam pipe radius and $V_{\max }$ is the largest desirable voltage to be applied to the steering dipoles. $V_{\max }$ is chosen to be as small as possible, to minimize beam disturbances. This method tends to apply smaller "kicks" using steering modules in more locations.

Our modeling results indicated that both methods would serve to steer the beam around the recirculator. We had intended to conduct experiments using these two algorithms to determine the optimum steering method. Unfortunately, the delay in completion of the recirculator confined our work in this area to computer simulations.

\section{Injector Pulse Modulators}

As we progressed in our beam transport experiments, we began to recognize the need for a more precise high potential ion source to produce a potassium ion beam in the existing transport line and bend. The recirculator injector pulse modulators must be capable of producing very precise voltage pulses in order to reduce the current modulation and achieve the required beam reproducibility. Initial beam control efforts on a completed recirculator will be focused on making corrections to the steering and acceleration waveforms on a pulse to pulse basis. Therefore, it is essential that the beam energy and timing from one pulse to the next are as nearly identical as possible. Modeling simulations have determined that errors greater than $0.1 \%$ in the flatness of the injector pulse can create intolerable beam energy deviations. Achieving these specifications has pushed the limits of present pulse power technology, including the methods used to measure the error in the modulator voltage pulse.

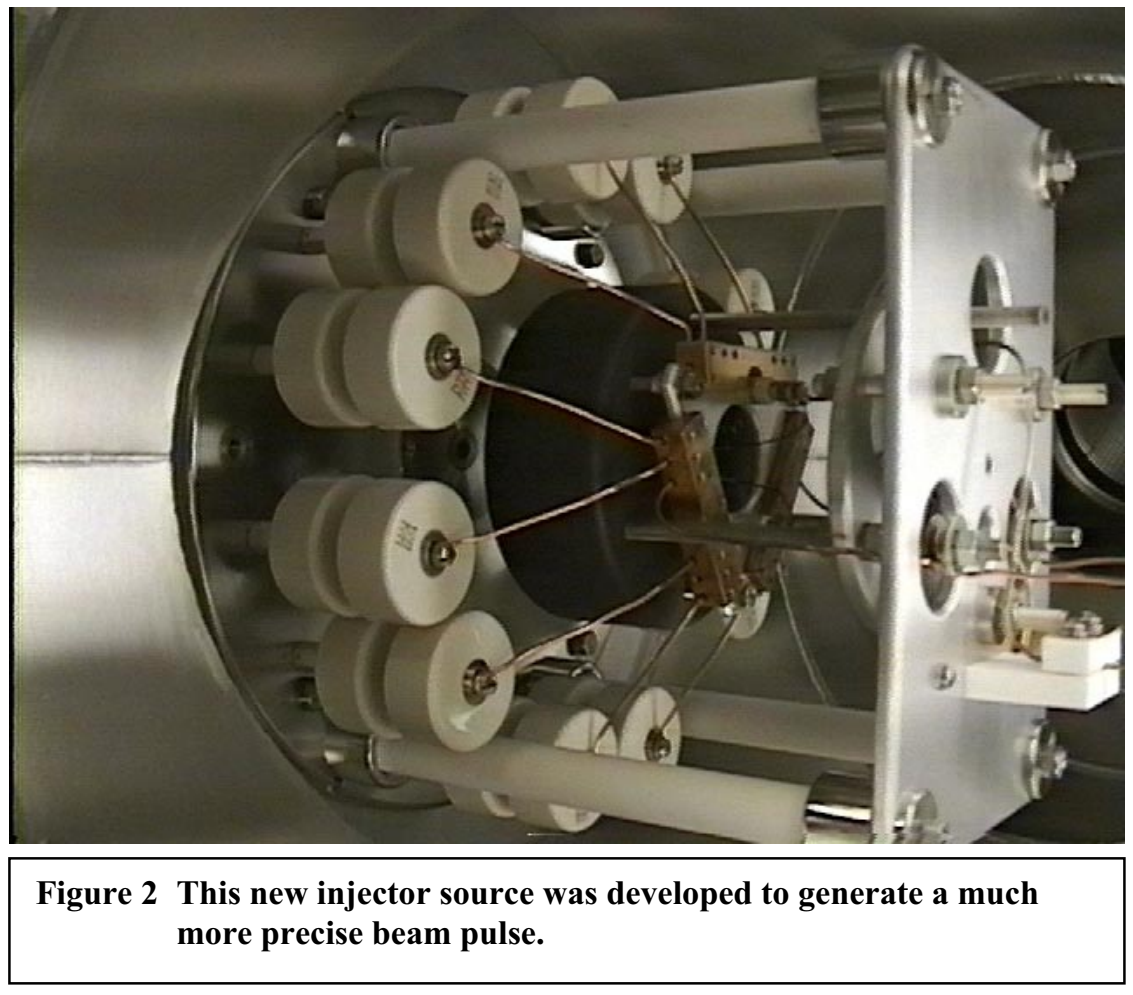


During FY97, we constructed and tested a new, higher voltage $(120 \mathrm{kV})$, higher reliability pulse generator (Figure 2), which surpassed the previous generator in several key parameters. The new generator produces a 5 microsecond pulse which is flat to less than $\sim 0.1 \%$. The rise and fall time of the pulse have an RC type shape which is now adjustable by simply modifying resistor values. A complete assessment and characterization of the injector performance, including beam measurements, was carried out in FY98. A full description, including detailed test results, for this injector source can be found in Reference [3].

\section{Dipole Pulser Development}

The completed recirculator will require 40 electrostatic dipoles, one in each half-lattice period (HLP), to bend the beam around the ring, as well as to provide beam position error correction in the X plane (the plane of the bend). As the beam is accelerated, the electric field necessary to keep the ions in a circular orbit increases with the ion energy. The dipoles must be driven such that the potential difference between their plates matches the energy of the beam as it passes through. The pulser output must therefore approximate a quadratically increasing function of time, ramping from $7 \mathrm{kV}$ to $27 \mathrm{kV}$ in approximately 228 microseconds. In addition, the opposing plates must be driven with voltages of opposite polarities so that the electric field at the point halfway between them is zero.

During FY97 a single polarity prototype pulse generator designed to meet the recirculator dipole requirements was built and tested in collaboration with researchers at LBNL. This pulse generator makes use of hard-tube pulsers and was capable of driving five dipoles (The charging current of the cable capacitance that connects the pulser to the dipoles limited the maximum number of dipoles that can be driven by one tube). Upon completion of the initial testing, the pulser was delivered to LLNL, where we carried out further evaluation during FY98.

Integration of the pulser with the recirculator and subsequent operations demonstrated the ability of the pulse power components to produce a programmable output pulse that could meet the most critical aspect of the design, namely, the voltage ramp of the required amplitudes.

Nevertheless, we also identified areas in which the pulser required specific modifications. For example, the prototype pulser was built as a standalone device, so its method for programming the output pulse shape did not lend itself readily to the recirculator's precise triggering requirements. Additionally, over continued operation the pulser's output transformers exhibited a tendency to overload and burn out.

The pulser was re-designed and re-packaged to modify the its waveform generation and control feedback elements, in order to integrate it into the existing recirculator timing, control, and diagnostics sub-systems, and to compensate for the effects of output loading and filtering. The basic pulsed power design of the original pulser was largely retained. The pulser was re-built to provide matching bipolar outputs, and to drive twice as many dipole plates (10 versus 5). Shot to shot repeatability was improved to $0.2 \%$ within a specified output range (up to $20 \mathrm{kV}$ at $35 \mathrm{~V} / \mathrm{s}$ slope). The timing and triggering circuits were re-worked to better integrate with the existing recirculator pulsed power and diagnostics systems. We conducted beam dynamics experiments using the modified pulser, which determined the relative effects of timing, voltage level, and output ripple on beam bending. A sample of the experimental results is shown in Figure 3, which illustrates that the pulser is performing as required to maintain the beam centroid within $0.7 \mathrm{~mm}$ of center. 


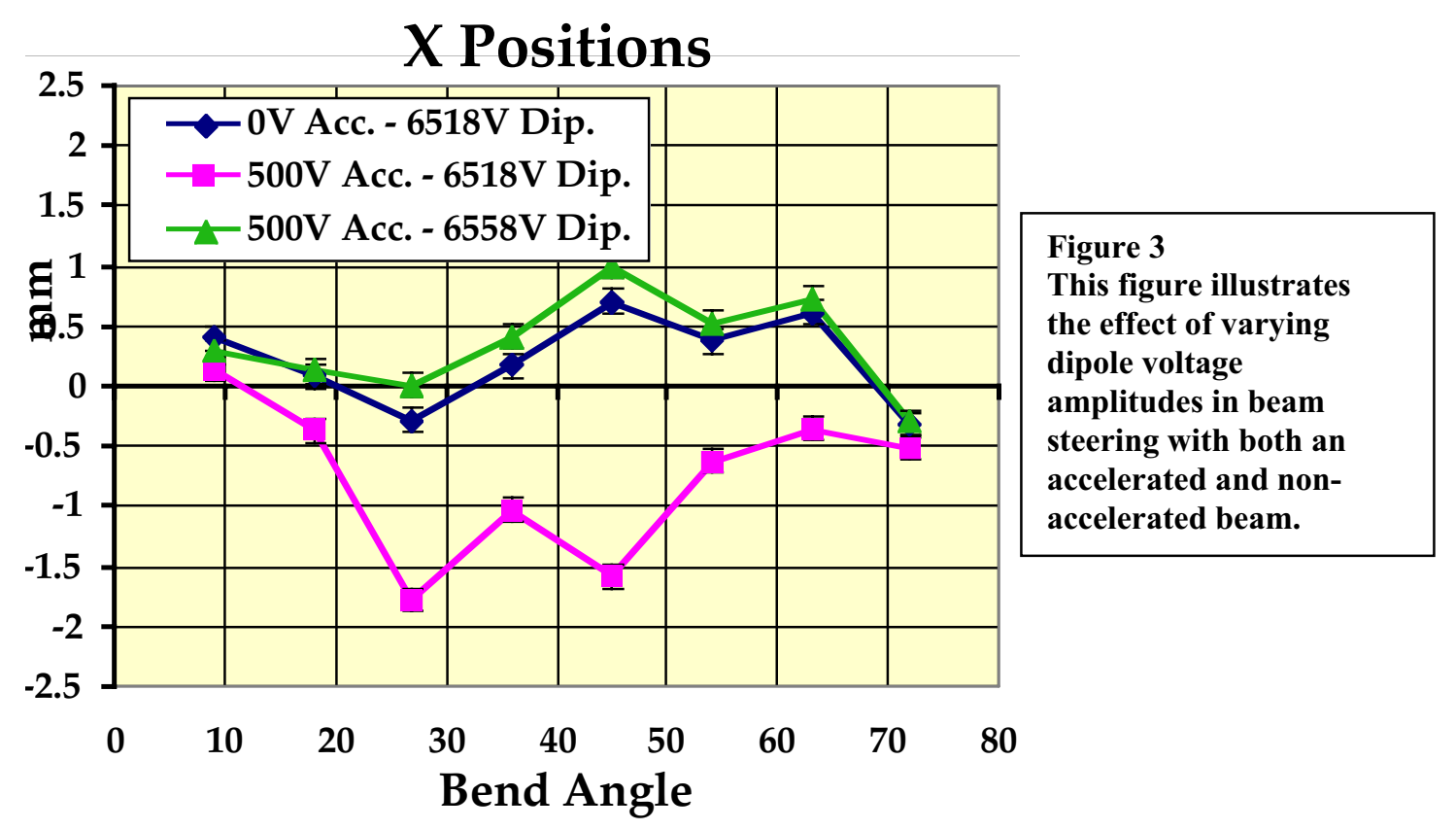

\section{Induction Cores and Accelerator Modulators}

The recirculator requires state-of-the-art, high repetition rate (up to $100 \mathrm{kHz}$ ) and highly variable pulse modulators for the induction cores. Pulse modulator specifications for the recirculator call for dynamic power control at a high bandwidth, the ability to produce a programmable pulse (fast rise-time waveforms with varying shapes, amplitudes, and widths) with a high degree of energy flatness and reproducibility $(<1 \%$ error) over the entire pulse length. This unique combination of performance specifications has required an intensive effort on the driver package design. The HIF Program sponsored two parallel modulator development efforts. Researchers in the LLNL Power Conversion Technologies (PCT) undertook one, which was part of the first two years of this LDRD. The other was part of a separate Small Business Innovative Research (SBIR) collaboration. In early FY99, the SBIR design was chosen for fabrication and installation on the recirculator. Nonetheless, the LLNL design effort produced some tangible results, which are reported below.

The baseline design of the LLNL modulators contains two feedback paths: fast local feedback with a single pole at $1.0 \mathrm{MHz}$, and a slower main feedback returned from the ferrite or Metglas ${ }^{\circledR}$ cores. The ratio of feedback from these sources is adjusted to maintain overall stability while still maintaining a specified degree of waveform fidelity between input and output signals. In FY97, one testbed modulator was constructed using a driver package fabricated from discrete components. Experimental results demonstrated a very good correlation between the measured modulator output voltage and the modeling data. However, simulations demonstrated that the level of overshoot exhibited by the prototype modulator was beyond the error tolerance needed to successfully achieve the desired accelerator performance.

The limiting factor in this design using discrete components (and the cause of the observed overshoot) was the inherent delays encountered due to the physical package configuration. The driver electronics package was converted to a hybrid microcircuit (HMC) in collaboration with Allied Signal Corporation. This reduced the physical size of the package, thereby reducing the 
impedances and increasing its bandwidth by shortening the signal paths, and decreasing the corresponding response time. Two types of prototype HMCs were delivered and tested in April 1997.

One type employed four parallel MOSFETs and associated drivers as switches while the other used them as linear control elements complete with feedback and proportional drive circuitry. The linear HMC version was installed and tested and demonstrated voltage regulation in excess of $200 \mathrm{~A}$ average current with peak currents of greater than $800 \mathrm{~A}$. Information gained as a result of the HMC tests contributed to the design of a new multi layer, stacked printed circuit board layout that promised higher fidelity and tighter regulation of modulator gap voltages, and a more effective and robust active core reset circuit. The selection of the SBIR design precluded further pursuit of these proposed design enhancements.

The recirculator acceleration cells contain two magnetic cores. The main core, which provides the beam acceleration, and the ear core, which provides the longitudinal compression necessary for minimizing beam emittance. It was determined that the ear cores (and to a lesser extent, the main cores) were only marginally capable of meeting the requirements of the LLNL design. The SBIR modulator design requires even higher volt-second core capacities. To increase this parameter without increasing core volume, higher performance materials were needed.

National Arnold was contracted to build both main and ear cores using Metglas ${ }^{\circledR} 2605 \mathrm{SC}$ material. The Metglas ${ }^{\circledR}$ material was coated with a very thin insulating layer then wound into its final form before undergoing a longitudinal annealing process. Once completed the cores were permeated with low viscosity silicon based oil. This final step improves the insulating properties of the core as well as reducing the magnastriction effects.

The SBIR design is based on a Miniature Inductive Adder concept. This approach allowed for a reduction in the number of induction modules needed for our small ring, and thus also to significant cost reductions in comparison to the FET technology employed by the LLNL design.

During FY99 we installed four stages of the SBIR modulator circuits and replaced ferrite cores

\section{HLP 5 ( $45 \mathrm{deg}$ )}

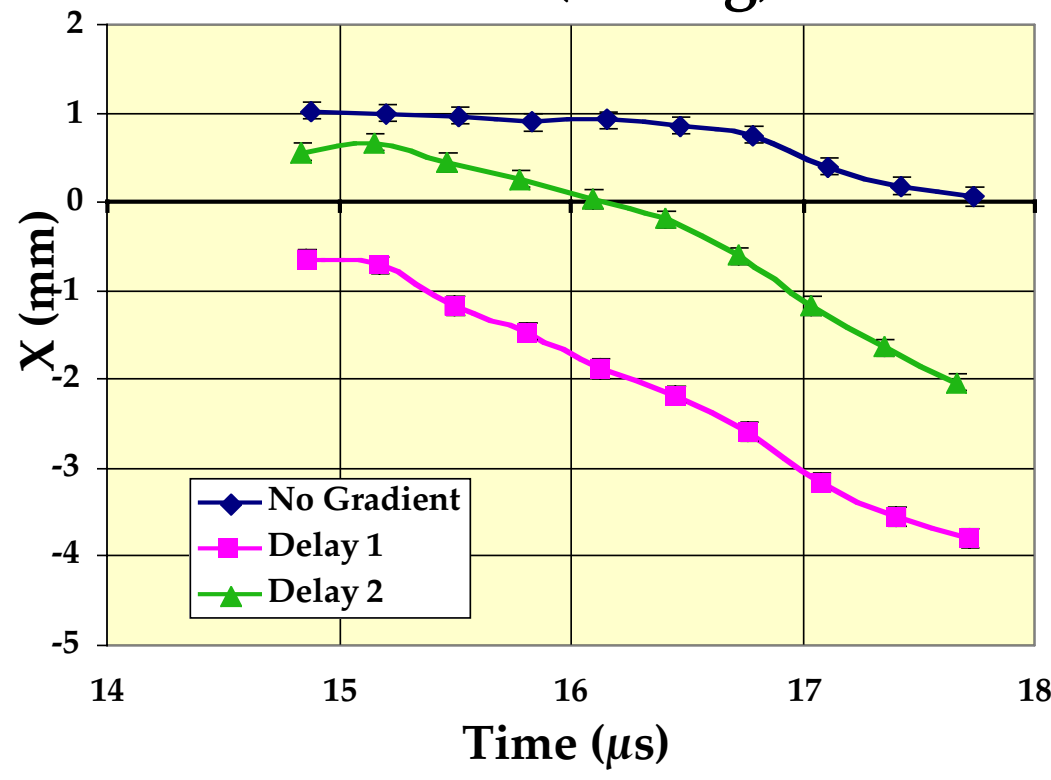

Figure 4 Plot of beam $X$ position versus time at one HLP for varying acceleration profiles. 
in some cells with the new Metglas ${ }^{\circledR}$ cores. We then ran a series of experiments to determine the accelerator cell and magnetic material characteristics. The cells containing ferrite material were able to sustain $500 \mathrm{~V}$ pulses for 5 _s, while the Metglas ${ }^{\circledR}$ cores sustained $1000 \mathrm{~V}$ pulses for 5 _s. The modulator pulse rise time was not fast enough to provide the necessary beam longitudinal confinement, due to larger than expected cell capacitance. The beam was maintained within 0.7 $\mathrm{mm}$ of the accelerator centerline throughout the 90-degree bend (see Figure 3). Figure 4 is a plot of the beam centroid transverse position as a function of time at HLP 5 located 45 degrees into the bend. The data was collected for the non-acceleration case (top trace) and with acceleration applied at two different delays from beam initiation. The position gradient indicates successful acceleration, with the beam tail containing more energy than the head.

\section{Insertion / Extraction Section}

Fast, affordable high voltage waveform generators are required to insert and extract the ion beam into and out of the recirculator ring. These waveform generators are likely to be based on a hardtube design (similar to the dipole pulse generators). These pulsers must rapidly (20 microseconds on insertion and 10 microseconds on extraction) ramp the voltage on one dipole plate from $+30 \mathrm{kV}$ to $-30 \mathrm{kV}$, which deflects or kicks the beam into or out of the recirculator ring.

This LDRD sponsored mechanical design and simulation efforts for the insertion / extraction section. Figure 5 illustrates one view of the completed design, which can be used in the fabrication and assembly for any further recirculator work.

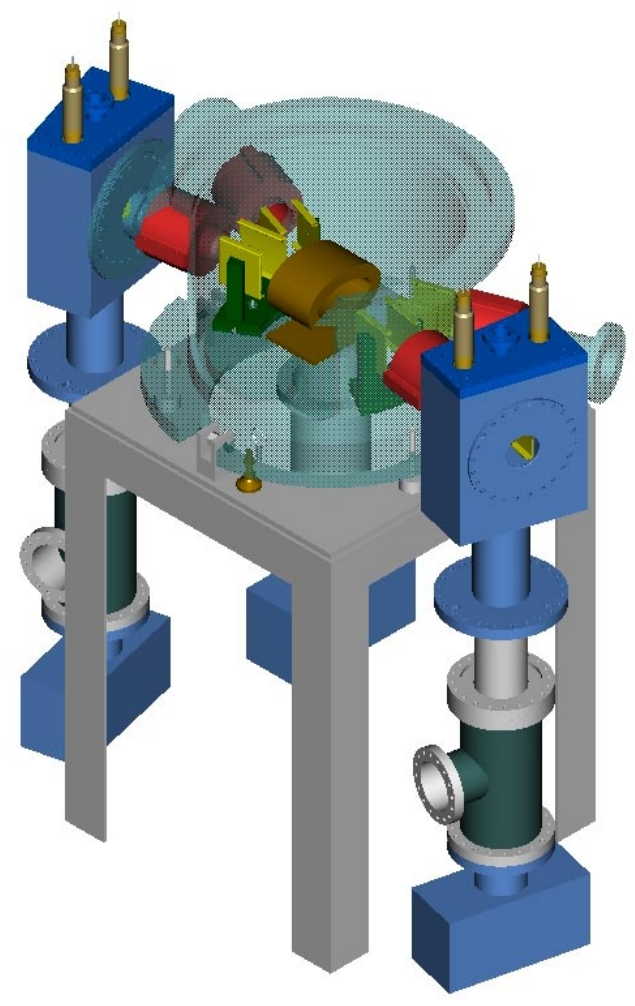

Figure 5 Insertion / extraction section design. 


\section{Conclusion}

While the accomplishments of this LDRD were different than those initially proposed, we were still able to materially increase our understanding of beam transport physics, make advances in induction core modulator technologies, and identify design enhancements for any future full recirculator. These developed capabilities and new knowledge are important to any acknowledged fusion driver scenario. Our increased understanding of the forcing components (acceleration and steering) will provide solid ground in which to analyze the transfer functions of a full recirculator, should one be realized. This in turn will allow for further effective research on adaptive beam control. We expect that this work will help establish a key role for LLNL in future projects employing advanced induction accelerators.

Future areas for development include implementing the beam timing and steering control system on fast, Digital Signal Processing (DSP) hardware for shot-to-shot control. An in-depth study of the beam transport physics in the presence of the control forcing functions will follow as the next essential element for concept validation. Finally, the results of future experiments can be used to refine our beam models, and apply more advanced control concepts to steer the beam from lapto-lap.

\section{Acknowledgments}

The authors would like to acknowledge Lou Reginato of Lawrence Berkeley National Laboratory for his work on the dipole pulser design. We would also like to acknowledge Dave Goerz, who contributed much to the work on the new injector pulser.

\section{References}

[1] Barnard, J. J., et. al., Study of Recirculating Induction Accelerators as Drivers for Heavy Ion Fusion, Lawrence Livermore National Laboratory, UCRL-LR-108095, September 1991.

[2] Sharp, W. M., Grote, D. P., Hernandez, M. A., Kamin, G. W., Steering Algorithms for a Small Recirculating Heavy Ion Accelerator, 12th International Symposium on Heavy Ion Inertial Fusion, September 24-27, 1997, submitted to Nuclear Instruments and Methods in Physics Research A, Lawrence Livermore National Laboratory, UCRL-JC-232229.

[3] Wilson, M., Goerz, D., Speer, R., Moal, R., Heavy Ion Fusion (HIF) Impulse Injector Design, Construction, and Checkout, Lawrence Livermore National Laboratory, UCRLID-130764.

[4] Sangster, T. C., et al., Status of Experiments Leading to a Small Recirculator, 12th International Symposium on Heavy Ion Inertial Fusion, September 24-27, 1997, submitted to Nuclear Instruments and Methods in Physics Research, UCRL-JC- 233095. 